\title{
On the Volume Infimum for Liquid Bridges
}

\author{
R. FINN and T. I. VOGEL
}

\begin{abstract}
We consider the "Carter conjecture", that any stable liquid bridge in zero gravity, joining two parallel plates separated by a distance $h$ and meeting each plate with constant angle, has volume greater then or equal to $h 3 / \pi$. We prove the conjecture in the case of the two contact angles being equal.

Key words: Capillarity, mean curvature, liquid bridge

AMS subject classification: $76 \mathrm{~B} 45,53 \wedge 10,49 \mathrm{Q} 10$
\end{abstract}

\section{Mise en scène}

Stability problems for liquid bridges that join parallel plates have been an active topic in the engineering literature for at least a century. More recently they have been studied from a rigorous mathematical point of view, in papers by Athanathanas [1], who considered the case of contact angle $\gamma=90^{\circ}$ on both plates, and by Vogel [11], who provided an independent discussion for that case and showed additionally [12] that with other choices of $\gamma$ a wide range of differing kinds of behavior must be expected. The studies by both authors assume vanishing gravitational field. A common feature of the configurations corresponding to any given contact angle (or pair of contact angles on the two plates) is that the bridge is stable if its volume $V$ is large enough, while if $V$ is continuously reduced from infinity then instability will occur when a critical value $V_{i}$ is crossed. In [1] and in [11] it was shown that when $\gamma=90^{\circ}$ the only stable configurations are circular cylinders, and $V_{i}=h^{3} / \pi$, where $h$ is the separation distance of the plates. This is half the volume occurring in the celebrated Rayleigh calculation [9], the factor of two appearing because of the particular boundary condition.

Independently of the above cited work and at about the same time, W. C. Carter [3] made extensive numerical stability calculations on liquid bridge configurations for different choices of $\gamma$, and observed that the greatest lower bound of drop volumes at which the bridge is stable always seemed to exceed the value that occurs when $\gamma=\pi / 2$ (circular cylinder). Vogel then considered the problem from a theoretical point of view [12], see also [10], and gave it informally the designation "Carter conjecture". The "conjecture" turned out to be of independent interest for the problem of designing "exotic" containers in such a way as to exclude stable liquid bridges joining the two end plates, see Finn [6]. In the present paper we intend to prove the statement completely for the case of equal angles $\gamma$ on the two plates. From the point of view of direct stability considerations, the basic step will be presented in $\S 5$, where we apply earlier results of Vogel in order to show that no rotationally symmetric configuration with an inflection in its meridian curve can be stable. In addition, every stable connected configuration is rotationally symmetric [11].

It is remarkable that these theorems are the only ways in which stability considerations are needed for the proof, the remaining steps being entirely geometric. We shall show that for any inflectionless equal angle configuration with $\rho=\pi V / h^{3} \leq 1$, 
a) the meridian curve must be an unduloid (see below), then

b) the unduloid must join two inflections at the plates, and finally

c) the unduloid must be a circular cylinder with $\rho=1$,

by showing that in any other case $\rho>1$, so that the volume would exceed the critical value for a circular cylinder. In this statement, the cylinder is viewed as a limiting configuration of approximating unduloids, with inflections at the limit points of those of the unduloids.

The underlying starting point for the entire theory is the observation that any equilibrium surface in the absence of gravity necessarily has constant mean curvature $H$ (see, e.g., [5], Chapt. 1). It thus suffices to consider solutions of the equation

$$
(r \sin \psi)_{r}=2 r H \quad \text {; }
$$

here $\psi$ is the angle made with the $r$-axis by a tangent to the meridian curve $u(r)$. At points where $\psi$ is an odd multiple of $\pi / 2,(1.1)$ is to be interpreted in the form

$$
\frac{\sin \psi}{r}-(\cos \psi)_{u}=2 H
$$

from this latter form we see immediately that the surface is either a circular cylinder of radius $1 / 2 \mathrm{H}$, or else the points of verticality on the meridian curve are isolated and not inflections, see Fig. 1.

If we normalize $\psi$ to be $\pi / 2$ at an "inner" vertical point $P:(a, 0)$, then the possible modes of behavior divide as follows, in terms of surfaces introduced originally by Delaunay [4] as roulades of the focal points of conics:

a) $H>0$ : the solution can be continued to form a periodic curve without double points, known as the unduloid, see Fig. 1a. Exactly two inflections appear in each period interval.

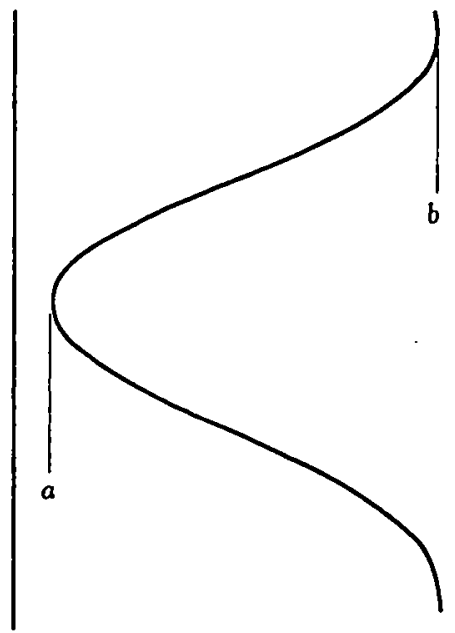

Figure 1a: Unduloid 
b) $H=0$ : we obtain the well-known catenoid, a minimal surface with the explicit representation

$$
r=a \cosh \frac{u}{a}
$$

In this case there is only the single (initial) vertical point, see Fig. 1b. There are no inflections and no double points.

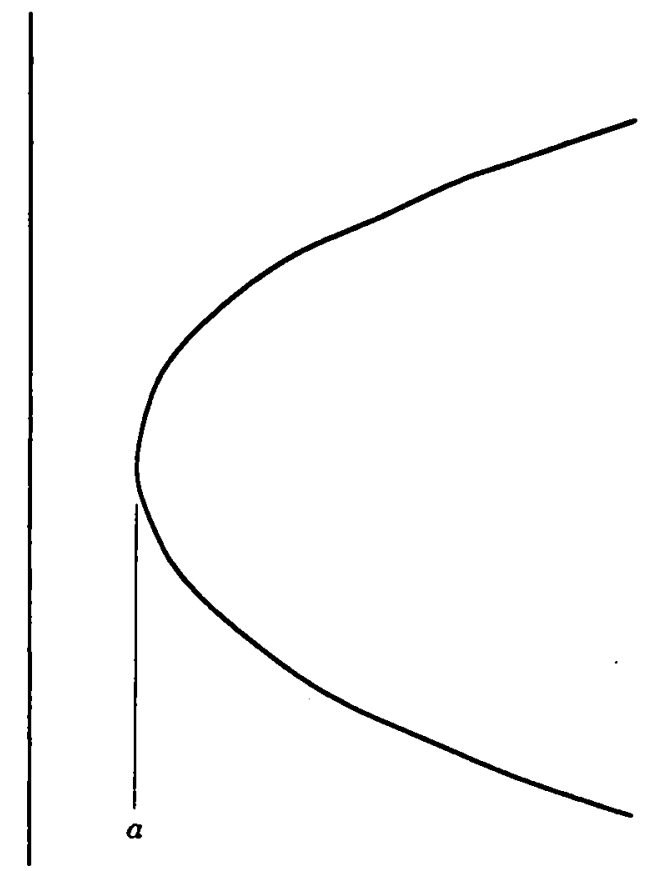

Figure 1b: Catenoid

c) $H<0$ : the continuation yields again a periodic curve, known as the nodoid. In this case there appear two double points in each period interval, but no inflections, see Fig. 1c.

One sees easily that these three cases encompass (up to rigid vertical displacement) the totality of solutions of (1.1), and thus, in view of Vogel's symmetry theorem, the totality of surfaces that can conceivably form physical bridges between two horizontal planes. Since (1.1) is formally invariant under similarity transformations, we can assume the planes at $u=0, h$, and we shall adjust the surfaces accordingly to achieve the prescribed contact angles.

As noted above, if $\gamma=\pi / 2$, the only stable solutions are circular cylinders, for which the infimum of stable volumes is $V_{i}=h^{3} / \pi$. We intend to show that any solution making equal angles on both planes and having no inflections between the planes bounds a larger volume. In the following two sections we show that the meridian curve of any solution can be assumed to be an unduloid, with inflections at the heights of the planes. 


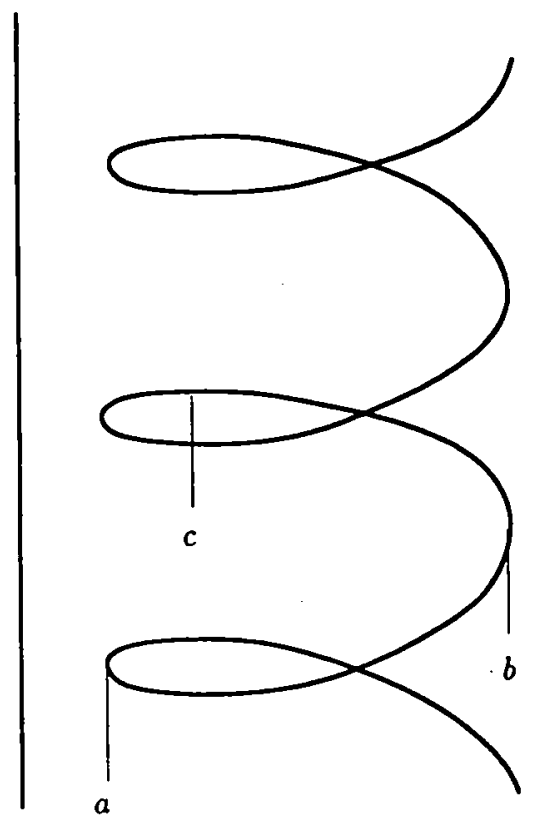

Figure 1c: Nodoid

\section{Exclusion of catenoids and nodoids}

The most general catenoid making the prescribed angles on the plates is situated symmetrically between $u=0$ and $u=h$ and has the form

$$
r=a \cosh \frac{u-h / 2}{a}
$$

the volume enclosed between the two planes is

$$
V=\frac{\pi a^{3}}{2}\left(\frac{h}{a}+\sinh \frac{h}{a}\right)
$$

We wish to show that for every $a, V$ exceeds $h^{3} / \pi$. Sctting $t=h / a$, it suffices to show that the function $f(t)=t+\sinh t-2 t^{3} / \pi^{2}$ is positive for positive $t$. We have

$$
f(0)=0, \quad f^{\prime}(t)=1+\cosh t-\frac{6}{\pi^{2}} t^{2}, \quad f^{\prime \prime}(t)=\sinh t-\frac{12}{\pi^{2}} t .
$$

Thus, $f^{\prime}(0)=2>0, f^{\prime \prime}<0$ in an interval $0<t<t_{0} \approx 1.1039$, with a single minimum at $t_{m} \approx 0.6458$, and $f^{\prime \prime}(t)>0$ for $t>t_{0}$. We find $f^{\prime \prime}\left(t_{m}\right) \approx-0.0936>-0.1$, and thus $f^{\prime}(t)>2-0.12=1.88$ in the interval $0<t<t_{0}$. We have proved

Lemma 2.1: Every catenoid situated symmetrically between the planes $u=0$ and $u=h$ bounds with those planes a volume exceeding $V_{i}$.

We exclude inner loops of nodoids by comparison with a suitable catenoid. At nonvertical points, a nodoid satisfies (1.1) with $H<0$. Again to achieve the prescribed 
data it must be situated symmetrically between the planes. Denoting by $P$ the common (inner) vertical point of the nodoid and the catenoid, we write the equation of the catenoid in the form

$$
(r \sin \phi)_{r}=0 \text {. }
$$

We select the segments of the two curves that lie above $P$, and find, under the normalization $\phi(P)=\psi(P)=\pi / 2$, that $\sin \psi-\sin \phi<0$ until the next vertical of the nodoid. Thus, the nodoid lies outside the catenoid between the two planes. We have proved

Lemma 2.2: Every inner nodoid loop situated symmetrically between the planes $u=0$ and $u=h$ bounds with these planes a volume exceeding $V_{i}$.

It remains to consider one of the outer loops of the nodoid, c.f. Fig. 1. On such a loop, (1.1) holds with $\psi=-\pi / 2$ at the outer vertical $r=b$. Denoting by $r=c$ the position of the horizontal points on the loop, we find $r \sin \psi=H\left(r^{2}-c^{2}\right)$, with $H=-b /\left(b^{2}-c^{2}\right)$, and thus

$$
2 H r=\sin \psi-\sqrt{\sin ^{2} \psi+4 H^{2} c^{2}} .
$$

Writing $\cos \psi=\sin \phi$ we find that an outer loop joining horizontal points is described by the range $-\pi / 2 \leq \phi \leq \pi / 2$, and (2.3) takes the form

$$
r=b\left\{\frac{k}{1+k} \cos \phi+\frac{1}{1+k} \sqrt{1-k^{2} \sin ^{2} \phi}\right\}
$$

with

$$
k=\frac{b^{2}-c^{2}}{b^{2}+c^{2}} .
$$

Since $d y / d r=\tan \psi=-\cot \phi$, we obtain from $(2.4)$

$$
\frac{d y}{d \phi}=b\left\{\frac{k}{1+k} \cos \phi-\frac{1-k}{\sqrt{1-k^{2} \sin ^{2} \phi}}+\frac{\sqrt{1-k^{2} \sin ^{2} \phi}}{1+k}\right\}
$$

and thus the height change between successive horizontal points is

$$
\Delta y=2 b\left\{\frac{k}{1+k}-(1-k) \mathrm{K}(k)+\frac{1}{1+k} \mathrm{E}(k)\right\}
$$

where $K(k)$ and $E(k)$ are complete elliptic integrals of the first and second kinds, respectively.

We find easily that for fixed $b$

$$
\frac{d}{d k}(\Delta y)=\frac{2 b}{1+k^{2}}\{1-\mathrm{E}(k)+(1+k) \mathrm{K}(k)\}>\frac{1}{1+k^{2}}>0
$$

and thus the height change decreases with decreasing $k$. In fact, since $E(0)=K(0)=$ $\pi / 2$, this height change tends to zero with $k$. Also, $H=-(1+k) / 2 b k$ becomes more 
negative with decreasing $k$, and a repetition of the comparison reasoning used above to exclude the inner loops of nodoids shows that two non-horizontal portions of outer loops with a common vertical do not otherwise intersect (even at the limiting horizontal points). Considering now a configuration bounded by two plates of separation $h$ and by a surface generated by outer loops of nodoids, we see that by decreasing (if necessary) $k$, and keeping $b$ constant, we can achieve a new configuration with smaller or equal volume, bounded by the same plates and by a nodoidal surface that meets the plates tangentially. It suffices to exclude surfaces with this structure.

For such surfaces we have $\Delta y=h$ in (2.7), and thus by (2.8) $b$ decreases with increasing $k$. In the limit as $k \rightarrow 1$, the configuration becomes a sphere of radius $h / 2$. From (2.7) we see that $b \rightarrow \infty$ when $k \rightarrow 0$ (since $\mathrm{E}(0)=\mathrm{K}(0)=\pi / 2$ ), and from (2.5) follows also $c \rightarrow \infty$. Thus either the sphere lies interior to all other surfaces of the family or else there will exist a particular such surface $\Sigma$, which contains the sphere but contacts it tangentially at some point $p$, as indicated in Fig. 2.

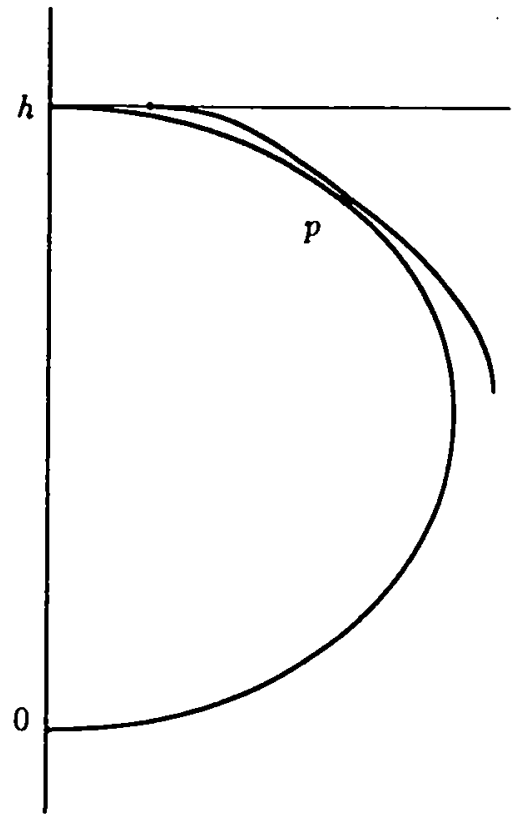

Figure 2: Exclusion of nodoid outer loop

Denoting by $H$ the mean curvature of $\Sigma$, we clearly have $H>-1 / b$, as otherwise the surfaces would have to cross. Denoting by $d$ the $r$-coordinate of $p$ and integrating (1.1) for both surfaces from $c$ to $d$, we obtain

$$
d\left(\sin \psi(d)-\sin \psi_{\Sigma}(d)\right)-c(-\sin \psi(c))=(H+1 / b)\left(d^{2}-c^{2}\right)>0
$$

and thus $\sin \psi(d)>\sin \psi_{\Sigma}(d)$, a contradiction to the assumed tangency at that point. Thus all surfaces of the family have volumes exceeding that of the sphere. But for the sphere we calculate easily $\pi V / h^{3}=\pi^{2} / 6>1$. Therefore, outer loops of nodoids are excluded. The only possibilities left for consideration are the unduloids. Since by 
Theorem 5.7 below every unduloid with equal angles on the plates and one or more inflections is unstable, consideration is reduced to unduloids symmetrically situated with respect to the plates, with no inflections between the plates. Throughout the next two sections, we shall have to distinguish two cases, designated by "thick" and "thin".

\section{Inflections}

We consider a symmetrically placed piece of unduloid with inflections outside the interval $0 \leq u \leq h$, as indicated in Fig. 3a (thick case) or in Fig. 3b (thin case).

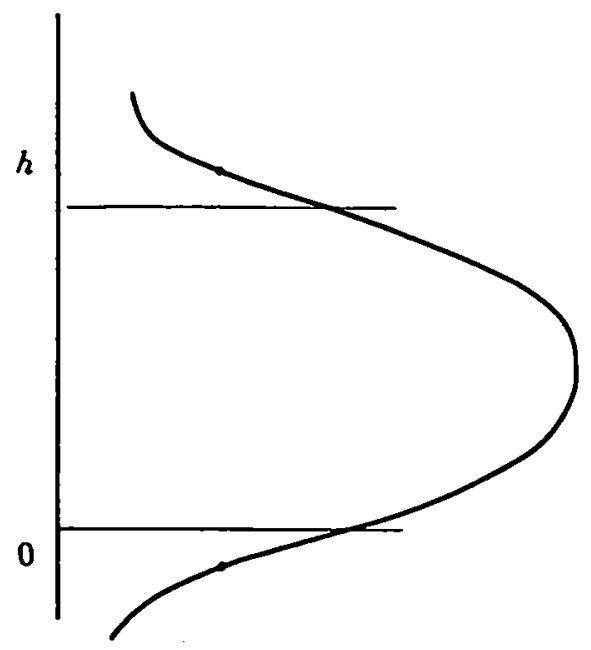

Figure 3a: Inflections outside interval (thick case)

In the former case, a similarity transformation (uniform contraction) centered at $(0, h / 2)$ creates a new unduloid, lying entirely interior to the region bounded between the original one and the two planes, and with inflections on the planes. The new unduloid clearly determines a smaller volume.

In the thin case, we denote by $\tau$ the height change from the inner vertical to the nearest inflection, and obtain

$$
\tau=a \frac{\mathrm{E}(k)-k}{1-k}, \quad k=\frac{b-a}{b+a}, \quad H=\frac{1}{a+b}
$$

cf the methods of $\$ 4$. Letting $b$ decrease toward $a$ we obtain a family of unduloids of increasing mean curvature, all vertical at a common (initial) point. Denoting the inclination angles of two meridian curves by $\phi$ and $\psi$, with $H_{\phi}<H_{\psi}$, we obtain immediately from the governing equation that $\sin \phi<\sin \psi$, and thus the surfaces yield decreasing volumes. As $b \rightarrow a$ the surfaces tend uniformly to a circular cylinder, and the inflections to a height $\tau_{0}=\pi a / 2$.

If $h>2 \tau_{0}$, then inflections will have appeared at the heights $0, h$ on some intermediate unduloid, and the original surface can be replaced by this new one with smaller volume. If $h<2 \tau_{0}$ we have since $\mathrm{E}(0)=\pi / 2$ that $h<\pi a$ and thus the volume of the limiting cylinder becomes $V_{0}=\pi a^{2} h \geq h^{3} / \pi$. We have proved 


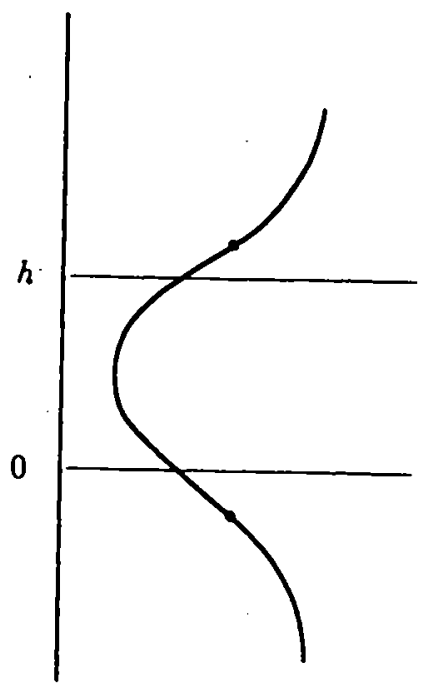

Figure 3b: Inflections outside interval (thin case)

Lemma 3.1: Every unduloid without inflections in the interval $[0, h]$ can be replaced, either by another one of smaller volume and infections exactly at $0, h$, or else by a cylinder of smaller volume $V_{0} \geq h^{3} / \pi$.

In view of Lemma 3.1, it will suffice to consider unduloids with inflections on the supporting planes $u=0, h$.

\section{Proof of the inequalities}

a) The thick case: In what follows we change notation somewhat from that of the preceding sections. Unduloids can be characterized analytically as rotation surfaces determined by solutions $r(r)$ of the equation system

$$
\begin{gathered}
(r \sin \psi)_{r}=2 r H \\
v_{r}=\tan \psi
\end{gathered}
$$

with verticals at $\left(r_{a}, v_{a}\right),\left(r_{b}, v_{b}\right)$ as indicated in Figs. 1a; 3a,b. The arcs can be reflected in either of the lines $v=v_{a}$ or $v=v_{b}$, so as to obtain a simply periodic solution curve, with an infinity of verticals and of inflections.

We are interested in the volume $V$ determined by a "bulge segment" between two inflections, as indicated in Fig. 3a. Let $h$ denote the change in the $v$ coordinate, between the inflections. We shall use the subscript $i$ to denote the value of a quantity at an inflection.

Lemma 4.1: In the thick case, therc holds $V \geq h^{3} / \pi$, with equality only for the circular cylinder. 
Proof: From (4.1) we find that on the initial lower segment of the curve, from $r_{i}$ to $r_{b}$, there holds

$$
r=\frac{\sin \psi+\sqrt{k^{2}-\cos ^{2} \psi}}{2 H}, \quad \psi_{i}<\psi \leq \pi / 2
$$

with

$$
k=\frac{r_{a}-r_{b}}{r_{a}+r_{b}}=\cos \psi_{i}, \quad H=\frac{1}{r_{a}+r_{b}} .
$$

We have also $r_{i}=\sqrt{r_{a} r_{b}}$.

The transformation

$$
\cos \psi=k \sin \phi, \quad 0 \leq \phi \leq \pi / 2
$$

yields $r=\left(k \cos \phi+\sqrt{1-k^{2} \sin ^{2} \phi}\right) / 2 H$ and, in view of (4.2),

$$
2 H \frac{\partial v}{\partial \phi}=-k \cos \phi-\sqrt{1-k^{2} \sin ^{2} \phi}
$$

from which

$$
H\left(v_{i}^{+}-v_{i}^{-}\right)=H h=k+\int_{0}^{\pi / 2} \sqrt{1-k^{2} \sin ^{2} \phi} d \phi=k+\mathrm{E}(k)
$$

where $E(k)$ is a complete elliptic integral of the second kind. From

$$
V=\pi \int_{0}^{\pi / 2} r^{2} \tan \psi \frac{\partial r}{\partial \phi} d \phi
$$

we now obtain by formal calculation

$$
\frac{4}{\pi} V=\frac{h^{3}}{(k+\mathrm{E}(k))^{3}}\left\{3 k-\frac{k^{3}}{3}+\frac{7+k^{2}}{3} \mathrm{E}(k)-\frac{4}{3}\left(1-k^{2}\right) \mathrm{K}(k)\right\},
$$

where

$$
\mathrm{K}(k)=\int_{0}^{\pi / 2} \frac{d \phi}{\sqrt{1-k^{2} \sin ^{2} \phi}}
$$

is a complete elliptic integral of the first kind.

The entire range of possibilities is encompassed in the range $0 \leq k \leq 1$. The value $k=0$ corresponds to a circular cylinder, $k=1$ to a sphere. Letting $V_{0}$ be the value in the (limiting) cylindrical case, we find $V_{0}=h^{3} / \pi$ and thus

$$
F(k) \equiv \frac{4(k+E)^{3}}{\pi h^{3}}\left(V-V_{0}\right)=3 k-\frac{1}{3} k^{3}+\frac{7+k^{2}}{3} E-\frac{4}{3}\left(1-k^{2}\right) \mathrm{K}-\frac{4}{\pi^{2}}(k+E)^{3}
$$

Our problem is thus reduced to showing that $F(k)>0$ in $0<k \leq 1$. 

that

We have clearly $F(0)=0, F(1) \approx 2.0911>0$. We observe next (cf [7], pp 73-76)

$$
\begin{gathered}
\frac{d \mathrm{E}}{d k^{2}}=-\frac{1}{2} \int_{0}^{\pi / 2} \frac{\sin ^{2} \phi}{\sqrt{1-k^{2} \sin ^{2} \phi}} d \phi \\
\frac{d \mathrm{~K}}{d k^{2}}=\frac{1}{2} \frac{1}{1-k^{2}}\left(\mathrm{~K}-\int_{0}^{\pi / 2} \frac{\sin ^{2} \phi}{\sqrt{1-k^{2} \sin ^{2} \phi}} d \phi\right), \\
k^{2} \int_{0}^{\pi / 2} \frac{\sin ^{2} \phi}{\sqrt{1-k^{2} \sin ^{2} \phi}} d \phi=\mathrm{K}-\mathrm{E}, \\
\int_{0}^{\pi / 2} \frac{\sin ^{2} \phi}{\sqrt{1-k^{2} \sin ^{2} \phi}} d \phi>\frac{1}{2} \mathrm{~K} .
\end{gathered}
$$

We calculate

$$
\begin{aligned}
\frac{d F}{d k^{2}}= & \frac{3}{2 k}-\frac{k}{2}+\frac{1}{3} \mathrm{E}-\frac{7+k^{2}}{6} \int_{0}^{\pi / 2} \frac{\sin ^{2} \phi}{\sqrt{1-k^{2} \sin ^{2} \phi}} d \phi+\frac{2}{3} \mathrm{~K} \\
& +\frac{2}{3} \int_{0}^{\pi / 2} \frac{\sin ^{2} \phi}{\sqrt{1-k^{2} \sin ^{2} \phi}} d \phi-\frac{6}{\pi^{2}} k-\frac{12}{\pi^{2}} \mathrm{E}-\frac{6 \mathrm{E}^{2}}{\pi^{2} k} \\
& +\frac{6}{\pi^{2}} k^{2} \int_{0}^{\pi / 2} \frac{\sin ^{2} \phi}{\sqrt{1-k^{2} \sin ^{2} \phi}} d \phi+\frac{12}{\pi^{2}} k \mathrm{E} \int_{0}^{\pi / 2} \frac{\sin ^{2} \phi}{\sqrt{1-k^{2} \sin ^{2} \phi}} d \phi \\
& +\frac{6}{\pi^{2}} \mathrm{E}^{2} \int_{0}^{\pi / 2} \frac{\sin ^{2} \phi}{\sqrt{1-k^{2} \sin ^{2} \phi}} d \phi .
\end{aligned}
$$

Since $E<\pi / 2$, we find $\frac{3}{2 k}-\frac{6}{k \pi^{2}} E^{2}>0$. Also, the coefficient of $k$ in $(4.16)$ is

$$
-\frac{1}{2}-\frac{6}{\pi^{2}}+\frac{12}{\pi^{2}} E \int_{0}^{\pi / 2} \frac{\sin ^{2} \phi}{\sqrt{1-k^{2} \sin ^{2} \phi}} d \phi>-\frac{1}{2}-\frac{6}{\pi^{2}}+\frac{6}{\pi^{2}} E K>1-\frac{6}{\pi^{2}}>0
$$

since, by the Cauchy-Bunyakovskii-Schwarz inequality,

$$
E K>\frac{\pi^{2}}{4}
$$

We thus obtain from the remaining terms in (4.16)

$$
\begin{aligned}
\frac{d F}{d k^{2}}> & \left(\frac{1}{3}+\frac{1}{6}-\frac{18}{\pi^{2}}\right) E+\left(-\frac{1}{6}+\frac{4}{3}-\frac{2}{3}+\frac{6}{\pi^{2}}\right) K \\
& +\left(-\frac{7}{6}+\frac{2}{3}+\frac{6}{\pi^{2}} E^{2}\right) \int_{0}^{\pi / 2} \frac{\sin ^{2} \phi}{\sqrt{1-k^{2} \sin ^{2} \phi}} d \phi \\
& >-.5738 \mathrm{E}+.8579 \mathrm{~K}>0
\end{aligned}
$$

by (4.15) and (4.17), since $K \geq E$. We conclude immediately that $F>0$ in $0<k \leq 1$, which proves the lemma. The function $F(k)$ is illustrated graphically in Figure $4 a$ 

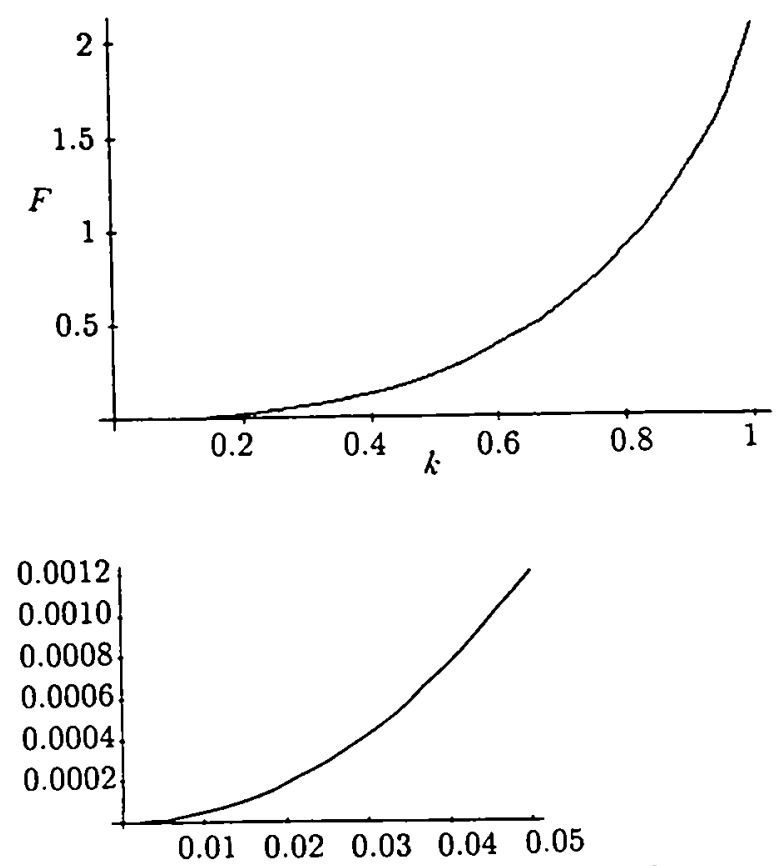

Figure 4a: The function $F(k)$ of $\S 4$

4b. Thin case: We study now the situation in which the considered arc lies closer to the axis than the inflections, as in Fig. 3b. In this case (4.3) must be replaced by

$$
r=\frac{\sin \psi-\sqrt{k^{2}-\cos ^{2} \psi}}{2 H}, \quad \psi_{i}<\psi<\pi / 2
$$

with

$$
k=\frac{r_{b}-r_{a}}{r_{b}+r_{a}}=\cos \psi_{i}
$$

The transformation

$$
\cos \dot{\psi}=k \sin \dot{\phi}, \quad 0<\phi<\pi / 2
$$

now yields

$$
r=\frac{-k \cos \phi+\sqrt{1-k^{2} \sin ^{2} \phi}}{2 H}
$$

and, in view of $d r / d \psi=\tan \psi$.

$$
H\left(v_{i}^{+}-v_{i}^{-}\right) \equiv H h=(-k+\mathrm{E}(k)) .
$$

For the volume

$$
V=2 \int_{0}^{\pi / 2} r^{2} \frac{d v}{d \phi} d \phi
$$


we obtain after some computation

$$
\frac{4}{\pi h^{3}} V=\frac{1}{(-k+E)^{3}}\left\{-3 k+\frac{1}{3} k^{3}+\frac{7+k^{2}}{3} E(k)-\frac{4}{3}\left(1-k^{2}\right) \mathrm{K}(k)\right\} .
$$

Thus, denoting by $V_{0}$ the volume of the (cylindrical) tube corresponding to $k=0$, and of height $h_{0}=\lim _{k \rightarrow 1} h(k)$ we are led to

$$
\begin{aligned}
\frac{4}{\pi}\left(\frac{V}{h^{3}}-\frac{V_{0}}{h_{0}^{3}}\right)=\frac{1}{(-k+\mathrm{E})^{3}} & \left\{-3 k+\frac{1}{3} k^{3}+\frac{7+k^{2}}{3} \mathrm{E}(k)-\frac{4}{3}\left(1-k^{2}\right) \mathrm{K}(k)\right. \\
& \left.-\frac{4}{\pi^{2}}(-k+\mathrm{E})^{3}\right\} \equiv \frac{1}{(-k+E)^{3}} Q(k) .
\end{aligned}
$$

It suffices to show that $Q(k)>0$ in $0<k<1$; the limiting case $k=1$ does not occur as a physical drop, and need not be considered.

The following relations, when derived in the sequence indicated, are easy formal consequences of the definitions:

$$
\begin{gathered}
\mathrm{E}(0)=\mathrm{K}(0)=\pi / 2 ; \quad \mathrm{E}(1)=1, \quad \mathrm{~K}(1)=+\infty \\
\frac{d \mathrm{E}}{d\left(k^{2}\right)}<0 ; \quad \frac{d^{2} \mathrm{E}}{d\left(k^{2}\right)^{2}}<0 \\
\mathrm{E}(k)>\frac{\pi}{2}-\left(\frac{\pi}{2}-1\right) k \\
\frac{d \mathrm{E}}{d k}=\frac{\mathrm{E}-\mathrm{K}}{k}<0 ; \quad \frac{\mathrm{K}}{d k}=\frac{1}{k}\left\{(\mathrm{E}-\mathrm{K})+\frac{k^{2}}{1-k^{2}} \mathrm{E}\right\} ; \quad \frac{d}{d k}(\mathrm{~K}-\mathrm{E})=\frac{k \mathrm{E}}{1-k^{2}} \\
\frac{\mathrm{E}(k)}{2} \ln \frac{1}{1-k^{2}}<\mathrm{K}-\mathrm{E}=\int_{0}^{k} \frac{t}{1-t^{2}} \mathrm{E}(t) d t<\frac{\pi}{4} \ln \frac{1}{1-k^{2}} \\
\frac{\pi}{2}\left(1-k^{2}\right)-k(1-k)<\mathrm{E}(k)-k<\frac{\pi}{8 k^{2}}\left[\left(1-k^{2}\right) \ln \frac{1}{1-k^{2}}+\left(1-k^{2}\right)\right]+(1-k) .
\end{gathered}
$$

The last inequality yields good information when $k$ is near unity, but is not satisfactory when $k$ is small. We improve it as follows:

$$
\begin{aligned}
\mathrm{E}(k)-k & =\int_{k}^{1}\left[\frac{\mathrm{K}(t)-\mathrm{E}(t)}{t}+1\right] d t=\int_{k}^{1} \frac{d t}{t} \int_{0}^{t} \frac{s \mathrm{E}}{1-s^{2}} d s+(1-k) \\
& =\int_{0}^{1-k} \frac{s \mathrm{E}}{1-s^{2}} d s \int_{0}^{1} \frac{d t}{t}+(1-k)<\frac{\pi}{2} \int_{0}^{1-k} \frac{s}{1-s^{2}} \ln \frac{1}{s} d s+(1-k)
\end{aligned}
$$

so that

$$
\mathrm{E}(k)-k<\frac{\pi}{4} \ln \frac{1}{1-k} \ln \frac{1}{k(2-k)}+\frac{\pi}{4} \int_{0}^{1-k} \frac{1}{s} \ln \frac{1}{1-s^{2}} d s+(1-k) .
$$


This estimate provides information at both endpoints; however, we do not require its full strength in what follows.

Using (4.24) and (4.28) we find

$$
Q(0)=Q(1)=0
$$

in the sense of limits from within the interval $0<k<1$. We have additionally

$$
\frac{d Q}{d k}=-3+k^{2}+k(\mathrm{E}+\mathrm{K})+\frac{1}{k}(\mathrm{E}-\mathrm{K})-\frac{12}{k \pi^{2}}(\mathrm{E}-k)^{2}(\mathrm{E}-\mathrm{K}-k)
$$

from which

$$
\frac{d Q}{d k}(0)=\frac{d Q}{d k}(1)=0
$$

and

$$
\begin{aligned}
Q^{\prime \prime} \equiv \frac{d^{2} Q}{d k^{2}}=2 k & +\mathrm{E}+\frac{1-k^{2}}{k^{2}}(\mathrm{~K}-\mathrm{E})-\frac{24}{k^{2} \pi^{2}}(\mathrm{E}-k)(\mathrm{K}-\mathrm{E}+k)^{2} \\
& +\frac{12}{k^{2} \pi^{2}}(\mathrm{E}-k)^{2}\left(\mathrm{E}-\mathrm{K}+\frac{k^{2}}{1-k^{2}} \mathrm{E}\right)
\end{aligned}
$$

from which

$$
Q^{\prime \prime}(0)=\frac{3 \pi}{2}-\frac{12}{\pi}>0, \quad Q^{\prime \prime}(1)=3>0 .
$$

From the above relations follows immediately that there are intervals about $k=0$ and $k=1$ in which $Q(k)>0$. We obtain a crude but adequate estimate for the size of these intervals by estimating each factor in each term individually. We start with the interval $0<k<0.1$ and consider the five terms on the right side of (4.33) in order, using the estimates (4.24) through (4.29). We find

$$
\begin{gathered}
2 k>0 \\
E(k)>E(.05)>1.569 \\
\left(1-k^{2}\right)>.9975 ; \quad \frac{K-E}{k^{2}}>\frac{E(.05)}{2} \frac{1}{k^{2}} \ln \frac{1}{1-k^{2}}>\frac{E(.05)}{2}>0.784 ;
\end{gathered}
$$

thus the third term of (4.33) is larger than 0.782 on $0<k<0.1$.

$$
\mathrm{E}-k<\frac{\pi}{2} ; \quad \frac{\mathrm{K}-\mathrm{E}}{k}<\frac{\pi}{4 k} \ln \frac{1}{1-k^{2}}<\frac{\pi}{4} \frac{k}{1-k^{2}}
$$

thus the fourth term of (4.33) is larger than -4.127 .

$$
\begin{gathered}
E-k>.9975 \frac{\pi}{2}-.0475 ; \quad \frac{E-K}{k^{2}}>-\frac{\pi}{4 k^{2}} \ln \frac{1}{1-k^{2}}>-\frac{\pi}{4} \frac{1}{1-k^{2}}>-0.788 \\
\frac{E}{1-k^{2}}>\mathrm{E}(k)>.9975 \frac{\pi}{2}+.0025>1.569
\end{gathered}
$$

thus the fifth term of (4.33) is larger than 2.192. Each of these estimates holds throughout the interval $0<k<0.05$; we conclude that $Q^{\prime \prime}>0.418$ throughout this interval. From (4.23) we compute $Q(0.05)>0.001$. 
The calculation near $k=1$ proceeds analogously, although we need a smaller interval for success. We obtain, for the interval $0.99<k<1$, that $2 k>1.98$ and $E(k)>E(1)=1$. Since $K>E$ we have the third term of (4.33) larger than 0 . Since $1-k^{2}<e^{-3}$ on the interval considered and $t \ln ^{\alpha}(1 / t)$ is increasing when $t<e^{-\alpha}$, each of the terms of the form $\left(1-k^{2}\right) \ln ^{\alpha}\left(1 /\left(1-k^{2}\right)\right)$ will be majorized at the lower end point $k=0.99$. Also, $1-k<1-k^{2}$. It follows that the fourth term of (4.33) is greater than -2.425 on the interval. One sees easily that the fifth term of (4.33) is positive. Adding the terms, we obtain $Q^{\prime \prime}>0.555$ on $0.99<k<1$, and thus $Q(k)>0$ on this interval. From (4.23), $Q(0.99)>0.00012$.
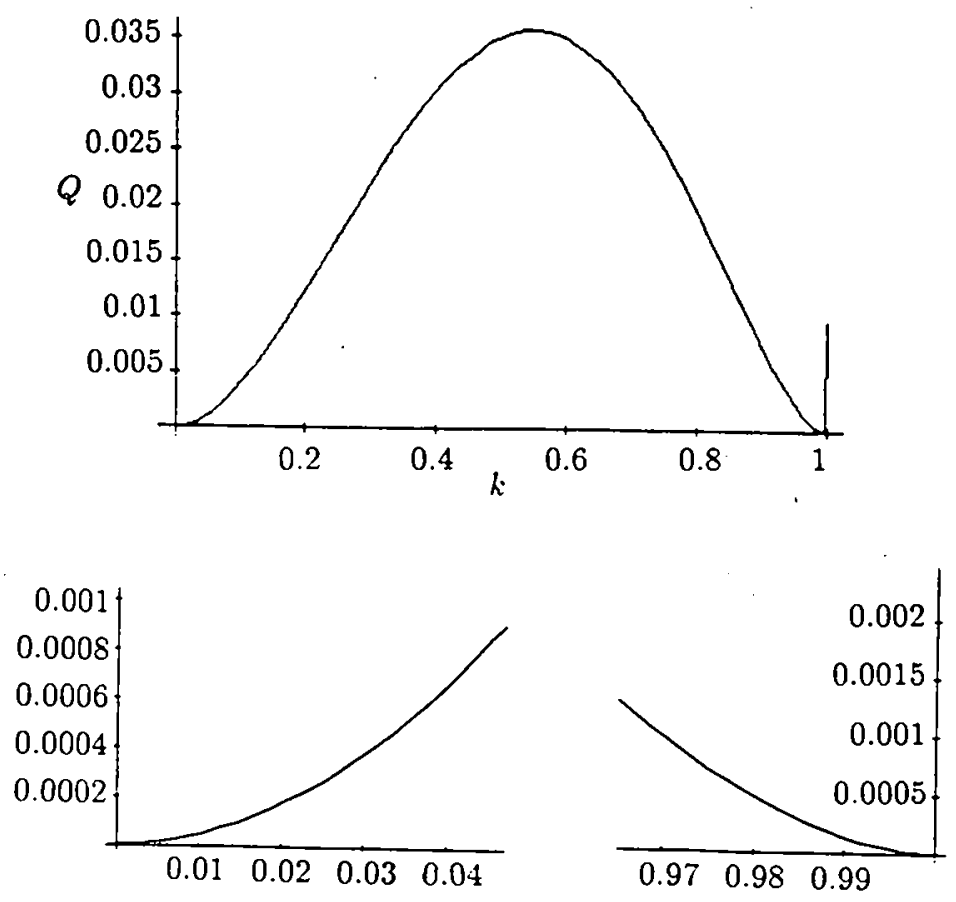

Figure 4b: The function $Q(k)$ of $\S 4$

The remaining interval $0.05 \leq k \leq 0.99$ is compact, and in that interval each of the terms in (4.23) can be estimated uniformly together with its derivatives. In Fig. $4 \mathrm{~b}$ is shown a formal graph of the function, obtained by standard computational procedures. We see that throughout the interior of the interval $Q$ exceeds its minimum at the end points. This assertion can be made rigorous by dividing the interval into a finite number of subintervals, in each of which the oscillation of $Q$ is less than its minimum at the end points. $Q$ can then be evaluated to sufficient accuracy at each endpoint of the subintervals so that the bounds will assure the positivity. We have proved

Lemma 4.2: In the thin case, there holds $V \geq h^{3} / \pi$, with equality only for the circular cylinder. 
Adjoining this information to Lemma 4.1, we have reduced the proof of the "Carter conjecture" to Theorem 5.7 , to be proved in the following section.

\section{Instability criteria}

This section will be devoted to deriving conditions under which a stationary liquid bridge must be unstable. In particular, it will be shown (Theorem 5.7 ) that in the case of equal contact angles, a profile with one or more inflections will be unstable. This along with the results of the previous sections will complete the proof of the Carter conjecture. We do not restrict ourselves to the case of equal contact angles until Lemma 5.6, however, and useful facts will be noted for unequal angles. We begin by summarizing the results from [11] which will be required. (The reader should be aware that in [11] the planes are being thought of as vertical, whereas in this paper they are horizontal.)

In contrast to the previous sections we will be considering the bridge surfaces nonparametrically, described by $r=f(u), u \in[0, h]$. This function must satisfy

$$
\begin{aligned}
\mathcal{M}(f) \equiv & \frac{1}{2}\left(\frac{f^{\prime \prime}}{\left(1+\left(f^{\prime}\right)^{2}\right)^{3 / 2}}-\frac{1}{f\left(1+\left(f^{\prime}\right)^{2}\right)^{1 / 2}}\right)=H \\
& f^{\prime}(0)=-\cot \gamma_{1} \\
& f^{\prime}(h)=\cot \gamma_{2}
\end{aligned}
$$

for some constant $H$, where $\mathcal{M}$ is the rotationally symmetric mean curvature operator and $\gamma_{1}$ and $\gamma_{2}$ are the contact angles with the planes $u=0$ and $u=h$. Condition (5.1) comes from the first variation, so that a solution to (5.1) is a stationary solution to the problem, and is the profile of a rotationally symmetric surface of constant mean curvature (the Delaunay surfaces, noted before). Not all stationary solutions are stable, however. For stability we must consider the second variation. A solution to (5.1) will be stable if the following two conditions are met.

1) The Sturm-Liouville problem

$$
\begin{gathered}
L(z) \equiv-\left(\frac{f z^{\prime}}{\left(1+\left(f^{\prime}\right)^{2}\right)^{3 / 2}}\right)^{\prime}-\frac{z}{f\left(1+\left(f^{\prime}\right)^{2}\right)^{1 / 2}}=\lambda z \\
z^{\prime}(0)=z^{\prime}(h)=0
\end{gathered}
$$

for $z(u)$ has precisely one negative eigenvalue, and

2) $f(u) \equiv f\left(u ; \epsilon_{0}\right)$ may be embedded in a smoothly parametrized family $f(u ; \epsilon)$ of solutions to (5.1) with $H=H(\epsilon)$, and $\gamma_{1}$ and $\gamma_{2}$ fixed, with $H^{\prime}\left(\epsilon_{0}\right) V^{\prime}\left(\epsilon_{0}\right)>0$, where $V(\epsilon)$ is the volume of the bridge corresponding to $f(u ; \epsilon)$.

A solution of (5.1) will be unstable if either condition is strictly violated, i.e., if (5.2) has two or more negative eigenvalues (it must always have at least one negative eigenvalue), or if $H^{\prime}\left(\epsilon_{0}\right) V^{\prime}\left(\epsilon_{0}\right)$ is negative.

It is natural to think that if a bridge is unstable, then extending that Delaunay surface (by considering planes with a larger separation and if necessary different contact angles) will only result in unstable bridges. However, due to the two different possible causes of instability, we were unable to prove this (the obvious idea of extending a

2 Analysis. Bd. 11. Heft 1 (1992) 
perturbation to which the smaller section of the unduloid is unstable runs. into the problem of requiring that the new perturbation be volume conserving). A useful result in this direction can be obtained, though. If the original bridge is unstable due to (5.2) having at least 2 negative eigenvalues, then taking a larger section of the unduloid will result in a bridge which is also unstable for the same reason. The proof will use Sturm-Liouville theory.

Lemma 5.1: Let $f$ be a solution to $\mathcal{M}(f)=H$, with no condition on contact angle. Suppose that $z$ solves $L(z)=0, z\left(u_{1}\right)=1, z^{\prime}\left(u_{1}\right)=0$, and that $z^{\prime}$ has $k$ roots in $\left(u_{1}, u_{2}\right)$. Then the number of negative eigenvalues of (5.2) on the interval $\left[u_{1}, u_{2}\right]$ is $k+1$.

Proof: This follows from the oscillation theorem for Sturm-Liouville equations. Using the notation of [2], we let $\theta(u ; \lambda)$ be the phase variable for (5.2), arising from the Prüfer substitution. The differential equation that $\theta$ satisfies is

$$
\frac{d \theta}{d u}=\left(\lambda+\frac{1}{f\left(1+\left(f^{\prime}\right)^{2}\right)^{\frac{1}{2}}}\right) \sin ^{2} \theta+\frac{\left(1+\left(f^{\prime}\right)^{2}\right)^{\frac{8}{2}}}{f} \cos ^{2} \theta .
$$

The initial condition is that $\theta\left(u_{1} ; \lambda\right)=\pi / 2$, and we are given that

$$
\frac{\pi}{2}+k \pi<\theta\left(u_{2} ; 0\right) \leq \frac{\pi}{2}+(k+1) \pi
$$

From Chapter 10, Section 7 of $[2], \theta(u ; \lambda)$ is strictly decreasing in $\lambda$ for fixed $u>0$. Those values of $\lambda$ for which $\theta\left(u_{2} ; \lambda\right)=\frac{\pi}{2}+n \pi$, with $n$ an integer, are eigenvalues of (5.3). From the Sturm oscillation theorem, $\lim _{\lambda \rightarrow-\infty} \theta\left(u_{2} ; \lambda\right)=0$, so that as $\lambda$ goes from 0 to $-\infty$, it will pass through $k+1$ eigenvalues, as desired

Lemma 5.2: Let $f$ be as in the previous lemma. Suppose that $\phi$ solves $L(\phi)=0$ and is not identically zero. If $\phi^{\prime}(u)$ has $k$ roots in $\left(y_{1}, y_{2}\right)$, then (5.2) has at least $k$ negative eigenvalues on $\left[y_{1}, y_{2}\right]$.

Proof: Let $z\left(u\right.$.) and $\theta$ be as defined in the previous lemma, and let $\theta^{*}(x)$ be the phase variable for $\phi$. We have that $\theta(u ; 0)$ and $\theta^{*}(u)$ solve $(5.3)$, and therefore cannot intersect unless they are identical. The fact that $\phi^{\prime}$ has $k$ zeroes implies that $\theta^{*}$ passes through $k$ odd multiples of $\frac{\pi}{2}$. Assume first that $\theta^{*}(0) \in\left(-\frac{\pi}{2}, \frac{\pi}{2}\right)$. Then we must have $\theta^{*}\left(y_{2}\right)>(k-1) \pi+\frac{\pi}{2}$. Since $\theta^{*}$ and $\theta(x ; 0)$ do not cross, it follows that $\theta\left(y_{2}, 0\right)>(k-1) \pi+\frac{\pi}{2}$. Lemma 5.1 will then apply, giving the desired result. If $\theta^{*} \notin\left[-\frac{\pi}{2}, \frac{\pi}{2}\right)$, we may add or subtract the appropriate multiple of $\pi$ to $\theta^{*}$. The result must still satisfy equation (5.3), and we may proceed as before

Theorem 5.3: Let $f(u)$ be the profile of an unduloid defined for all $u$. Suppose that the Sturm-Liouville problem for the operator $L$ defined in (5.2) with boundary conditions $z^{\prime}\left(u_{1}\right)=z^{\prime}\left(u_{2}\right)=0$ has more than one negative eigenvalue. Suppose that $y_{1} \leq u_{1}<$ $u_{2} \leq y_{2}$. Than the Sturm-Liouville problem with boundary conditions $z^{\prime}\left(y_{1}\right)=z^{\prime}\left(y_{2}\right)=$ 0 has more than one negative eigenvalue.

Proof: Let $z(u)$ solve $L(z)=0$ with $z\left(u_{1}\right)=1, z^{\prime}\left(u_{1}\right)=0$. By Lemma 5.1, $z^{\prime}$ must have a second zero before $u_{2}$. Using Lemma 5.2 with $z$ in place of $\phi$, the result follows 
Corollary 5.4: A bridge whose profile has two vertical points is unstable.

Proof: From the appendix of [12] we know that if $f^{\prime}\left(u_{1}\right)=0$ and $f^{\prime}\left(u_{2}\right)=0$ then the Sturm-Liouville problem for $L$ with boundary conditions $z^{\prime}\left(u_{1}\right)=z^{\prime}\left(u_{2}\right)=0$ has at least two negative eigenvalues. The result now follows from Theorem 5.3

Corollary 5.5: A bridge whose profile has two or more inflections is unstable.

Proof: It is straight-forward to verify that $L\left(f^{\prime}\right)=0$. Since $f^{\prime \prime}$ has at least two zeroes, the result follows from Corollary 5.2

We now restrict ourselves to drops with equal contact angles, to obtain the stability results required to prove the Carter conjecture. From [12] we know that if there are no inflections in the profile curve then $\lambda_{0}<0<\lambda_{1}$, and from Corollary 5.5 we know that if there are two inflections then $\lambda_{1}<0$. We must now deal with the remaining case of one inflection. We first need a technical lemma.

Lemma 5.6: Suppose that $u_{1}$ and $u_{2}$ satisfy $f^{\prime}\left(u_{1}\right)=-f^{\prime}\left(u_{2}\right)=\cot \gamma$ (where $f$ is the profile of an unduloid), and that $f^{\prime \prime}=0$ exactly once in $\left(u_{1}, u_{2}\right)$. Then

$$
u_{2}-u_{1}=\int_{0}^{\pi} \sqrt{a^{2}-c^{2} \cos ^{2} \theta} d \theta-2 a \cos \gamma,
$$

where $a$ and $c$ are the standard quantities for the ellipse generating $f$.

Proof: There are two cases to consider: $\gamma<\pi / 2$ and $\gamma>\pi / 2$. First assume that $\gamma<\pi / 2$. Let $\xi_{1}$ be the $u$ coordinate of the vertical point of the profile curve between $u_{1}$ and $u_{2}$, and let $\xi_{2}$ be the $u$ coordinate of the next vertical point after $\xi_{1}$ (the geometry of the unduloid forces $\xi_{2}$ to be greater than $u_{2}$ ). Since

$$
u_{2}-u_{1}=\left(\xi_{2}-\xi_{1}\right)-\left(\xi_{2}-u_{2}\right)+\left(\xi_{1}-u_{1}\right),
$$

we will need to compute each quantity on the right of (5.5).

$\xi_{2}-\xi_{1}$ is half the arclength of the generating ellipse, giving the integral in (5.4). The other two terms will be computed using formulas (3.10) and (3.11) from [12], for inclination angles 0 and $\pm \alpha$ (where $\alpha=\gamma-\pi / 2<0$ ) (the inclination angle is the angle the tangent to the meridional curve makes with a vector parallel to the $u$ axis, and is $\pi / 2$ minus the angle $\psi$ of Section 1 ). We shall assume that $f^{\prime \prime}\left(u_{1}\right)<0$ (otherwise we could reflect the unduloid across the line $\left.u=\left(u_{1}+u_{2}\right) / 2\right)$.

For $\xi_{1}-u_{1}$, instead of going from $u_{1}$ (where the inclination angle is $\alpha$ ) to $\xi_{1}$ (where the inclination angle is 0 ), we will go from $\xi_{1}$ to the first point beyond $\xi_{1}$ which has inclination angle $-\alpha$. By the symmetry of the unduloid, this is the same distance, and it has the virtue of telling us exactly what $R$ is in formula (3.10) of [12]: We obtain

$$
\xi_{1}-u_{1}=\frac{1}{2 H} \int_{0}^{-\alpha} \cos t-\frac{\cos ^{2} t}{\sqrt{\cos ^{2} t+4 R H+4 R^{2} H^{2}}} d t .
$$

Now, $R$ in the above formula is the radius at $\xi_{1}$ and is therefore $a-c$, and $H$ is $-1 /(2 a)$. Using these in the above equation yields

$$
\xi_{1}-u_{1}=-a \int_{0}^{-\alpha} \cos t-\frac{\cos ^{2} t}{\sqrt{\cos ^{2} t+\frac{c^{2}}{a^{2}}-1}} d t .
$$


Similarly,

$$
\xi_{2}-u_{2}=\frac{1}{2 H} \int_{0}^{\alpha} \cos t+\frac{\cos ^{2} t}{\sqrt{\cos ^{2} t+4 R H+4 R^{2} H^{2}}} d t
$$

where $R$ is now the radius at $\xi_{2}$ and is therefore $a+c$. Thus

$$
\xi_{2}-u_{2}=-a \int_{0}^{\alpha} \cos t+\frac{\cos ^{2} t}{\sqrt{\cos ^{2} t+\frac{c^{2}}{a^{2}}-1}} d t .
$$

From (5.6) and (5.7) we obtain an expression for $\left(\xi_{1}-u_{1}\right)-\left(\xi_{2}-u_{2}\right)$ :

$$
\begin{aligned}
&\left(\xi_{1}-u_{1}\right)-\left(\xi_{2}-u_{2}\right)= \\
& \quad a \int_{0}^{\alpha} \cos t-\frac{\cos ^{2} t}{\sqrt{\cos ^{2} t+\frac{c^{2}}{a^{2}}-1}} d t+a \int_{0}^{\alpha} \cos t+\frac{\cos ^{2} t}{\sqrt{\cos ^{2} t+\frac{c^{2}}{a^{2}}-1}} d t
\end{aligned}
$$

which is of course $2 a \sin \alpha=-2 a \cos \gamma$, completing the proof in the case $\gamma<\pi / 2$. The proof in the other case is similar and is omitted

Theorem 5.7: $A$ liquid bridge with equal contact angles and one or more inflections has $\lambda_{1}<0$ (and is therefore unstable).

Proof: The only case not yet dealt with is when $f$ has exactly one inflection point in $(0, h)$. In this case $f$ must be an unduloid (since nodoids and catenoids have no inflections). Assume that for this $f$ we have $\lambda_{1}>0$. The first step is to show that this implies the existence of a liquid bridge with equal contact angles with $\lambda_{1}=0$ and with one interior inflection.

Considering the unduloid of which $f$ is a section, we may extend $f$ to be defined for all $u$. Given an $u_{0}$, consider the function $z$ which solves $L(z)=0, z\left(u_{0}\right)=1$, and $z^{\prime}\left(u_{0}\right)=0$. We will define the point conjugate to $u_{0}$ to be the first $u$ value larger than $u_{0}$ for which $z^{\prime}(u)=0$. Denote this conjugate point by $\mathcal{C}\left(u_{0}\right)$. It is known [12] that a profile having contact angles $\frac{\pi}{2}$ will have $\lambda_{1}<0$ if $f$ is not constant. Thus (using Lemma 5.1) for our specific unduloid $f$, if $f^{\prime}\left(u_{0}\right)=0, \mathcal{C}\left(u_{0}\right)$ will be less than the next zero of $f^{\prime}$.

The assumption on $f$ amounts to assuming that $\mathcal{C}(0)>h$, in other words that $\mathcal{C}(0)$ is greater than the first root of $f^{\prime}(u)-\cot \gamma_{0}=0$, where $\gamma_{0}$ is the contact angle for the original unduloid. By continuity, therefore, as the contact angle varies from $\frac{\pi}{2}$ to $\gamma_{0}$, there will be an angle $\gamma_{1}$ such that for the value $u_{1}$ solving $f\left(u_{1}\right)=-\cot \gamma_{1}$, we have that $\mathcal{C}\left(u_{1}\right)$ is the first root of $f^{\prime}(u)-\cot \gamma_{1}=0$ greater than $u_{1}$. Therefore, for $\gamma_{1}$, we have that $(5.2)$ has $\lambda_{1}=0$ on the interval $\left[u_{1}, \mathcal{C}\left(u_{1}\right)\right]$. By translating and rescaling the interval $\left\{u_{1}, \mathcal{C}\left(u_{1}\right)\right]$ to $[0, h]$, we may assume that we have a function $f$ with $f^{\prime}(0)=-f^{\prime}(h)=\cot \gamma$, for some $\gamma$, that $f$ has exactly one inflection point in $(0, h)$, and that (5.2) has $\lambda_{1}=0$ for this $f$. We may also assume, by reflecting the graph of $f$ across the line $u=h / 2$ if necessary, that $f^{\prime}(u) \neq \cot \gamma$ for $u \in(0, h)$.

We will now see that this is impossible. The idea of the proof is similar to the one used in the appendix of [12], in that we will exhibit a specific function $z$ satisfying $L(z)=$ $0, z^{\prime}(0)=0$, but $z^{\prime}(h) \neq 0$. Let $a_{0}, b_{0}$, and $c_{0}$ be the standard quantities for the ellipse generating $f$. From Lemma 5.6 we have that $h=\int_{0}^{\pi} \sqrt{a_{0}^{2}-c_{0}^{2} \cos ^{2} \theta} d \theta-2 a_{0} \cos \gamma$. 
We now consider unduloids with the same mean curvature (hence the same value for a), but with differing $c$ 's. We translate these to have derivative equal to $\cot \gamma$ at $u=0$. To be more precise, let $g(u ; c)$ be the family of solutions of $\mathcal{M}(g)=-1 / 2 a_{0}$ with $g^{\prime}(0 ; c)=\cot \gamma$ for all $c$. Then as in [12], $L\left(g_{c}\left(0 ; c_{0}\right)\right)=0$ and $g_{c}^{\prime}\left(0 ; c_{0}\right)=0$. The proof that $g^{\prime}\left(h ; c_{0}\right) \neq 0$ is essentially the same as the proof of Theorem A.1 in the appendix of [12]. Define $\delta(c)$ to be the difference between $h$ and the second positive $u$ value satisfying $f^{\prime}(u)=-\cot \gamma$, so that

$$
\delta(c)=\int_{0}^{\pi} \sqrt{a_{0}^{2}-c^{2} \cos ^{2} \theta} d \theta-2 a_{0} \cos \gamma-h
$$

and $\delta\left(c_{0}\right)=0$. As in [12],

$$
g_{c}^{\prime}\left(h ; c_{0}\right)=-\delta^{\prime}\left(c_{0}\right) g^{\prime \prime}\left(h ; c_{0}\right)
$$

Neither of the quantities on the right of the above equation can be zero, so that $g_{c}^{\prime}\left(h ; c_{0}\right) \neq 0$. But this proves that 0 cannot be an eigenvalue of $L$, since the eigenfunction $z_{1}(x)$ would satisfy the same ordinary differential equation and the same initial conditions as $g_{c}(u) / g_{c}(0)$.

\section{Appendix. Characterization of the family of inflectionless pro- files}

Since we have seen that no stable drop with equal contact angles can have an inflection, it seems appropriate at this time to describe the family of inflectionless profiles (for equal contact angles), expanding on results of [12]. There it is shown that the family $\mathcal{F}$ of inflectionless profiles is connected, can be parametrized by $H$, and for large volumes the profiles are uniformly close to arcs of circles. We first deal with equal contact angles greater then $\pi / 2$.

Theorem A.1: For $\gamma>\pi / 2$, if $H>2 \cos \gamma$ the inflectionless profile with mean curvature $H$ is a nodoid ( $2 \cos \gamma$ is the mean curvature of the sphere with the appropriate contact angles), and if $H<2 \cos \gamma$ the profile is an unduloid.

Proof: The parametric equations for the profiles derived in [12] apply to both nodoids and unduloids. To tell which we have, we look for the possibility of an inflection if the curve continues (nodoids have no inflections, unduloids must have inflections), although of course we don't have an inflection on the interval $(0,1)$. If $\phi$ is the inclination angle, then using the explicit formula for $y_{0}(\phi)$ in [12], Equation (3.1), we find that

$$
\frac{d \phi}{d s}=\frac{2 H \sqrt{\cos ^{2} \phi+4 R H \sin \gamma+4 R^{2} H^{2}}}{\cos \phi+\sqrt{\cos ^{2} \phi+4 R H \sin \gamma+4 R^{2} H^{2}}}
$$

Here $R$ is the wetted radius on the plane $x=0$. If $\phi$ can get all the way to $\pi / 2$ without an inflection occurring, we must have a nodoid. (A.1) tells us that if $4 R H \sin \gamma+4 R^{2} H^{2}$ is positive, then no inflection occurs, but if $4 R H \sin \gamma+4 R^{2} H^{2}$ is negative, an inflection occurs before $\phi$ gets to $\pi / 2$ (once an inflection occurs, we may no longer use $y_{0}$ (which is for profiles of convex drops) and the derivation for (A.1) no longer applies). 
We are interested in the sign of $R H(\sin \gamma+R H)$, where $R$ is always positive and $H$ is always negative. In the proof of Theorem 4.2, [12], it is shown that $d(R H) / d H<0$ throughout the family of uninflected convex drops. For the sphere one easily checks that $R H=-\sin \gamma$. Thus for $H$ between $2 \cos \gamma$ and $\cos \gamma, R H(\sin \gamma+R H)>0$ and the profile is a section of a nodoid, and for $H<2 \cos \gamma$ the profile is a section of an unduloid.

We can now describe the family of uninflected profiles for equal contact angles greater than $\pi / 2$. The large volume limit is a piece of a cylinder whose mean curvature is easily calculated to be $\cos \gamma(<0)$. From Theorem A.1, the large volume profiles which are close to arcs of circles are sections of nodoids, and as the mean curvature decreases so does volume until we reach another circular arc (which is the profile of a sphere). As we continue reducing mean curvature, we pass through a series of unduloids (with volume still decreasing) until we finally reach an unduloid with inflections on both boundaries, at which point there is a bifurcation to a symmetric family of unduloids with two inflections and two families of one-inflection unduloids (proven in [10]). There are no more inflectionless unduloids, since the parameter set of $H$ 's for $\mathcal{F}$ is an interval [12]. The fact that volume is decreasing throughout is Theorem 4.2 of [12]

We now turn our attention to the case $\gamma<\pi / 2$, so that we deal with concave rather than convex drops. The large volume limit is again an arc of a circle with $H$ approaching $\cos \gamma(>0)$.

Theorem A.2: For $\gamma<\pi / 2$, if $H>0$ the uninfiected profile is a section of a nodoid, and if $H<0$ the uninflected profile is a section of an unduloid.

Proof: Again we look for the appearance of inflections on the continuation of the profile. Since $y \frac{d \phi}{d s}=\cos \phi+2 H y$, we want to see whether

$$
\sqrt{\cos ^{2} \phi-\sin ^{2} \gamma+(2 R H+\sin \gamma)^{2}}=\cos \phi+2 H y_{i}
$$

can get to zero. (For a concave drop we use the explicit expression for $y_{i}$ in [12], (3.6).) If $H>0$ the expression in (A.2) cannot be zero for any $\phi$, so we must have a piece of a nodoid. For $H<0$, we must have $0<2 R H+\sin \gamma<\sin \gamma$, where the first inequality comes from the sign of $\frac{d \phi}{d s}$ on the boundary plane. But then for $\phi=\pi / 2$, $\cos ^{2} \phi-\sin ^{2} \gamma+(2 R H+\sin \gamma)^{2}<0$, so we have an inflection before $\phi=\pi / 2$. Thus in this case the profile is an unduloid

The family of uninflected drops in the case $\gamma<\pi / 2$ therefore behaves as follows: in the large volume limit we have nodoids which are close to a circular arc with $H$ close to $\cos \gamma(>0)$. As $H$ decreases through zero, we pass through the nodoids, and at $H=0$ the profile is a catenary. As $H$ decreases further the profiles are unduloids, until again we reach the limit of the family when inflections appear on the boundary, with the same sort of bifurcation as before. We can no longer say that volume decreases as mean curvature decreases through the inflectionless family, however. It has bcen observed numerically that for $\gamma<\gamma_{0} \approx 31.14^{\circ}$ the volune is not decreasing throughout the family ([12]), and Langbein ([8]) has derived an transcendental expression of which $\gamma_{0}$ is a root. Moreover, it is conceivable that $V(H)$ has more than one local minimum 
even for $\gamma>\gamma_{0}$, which would imply that the family of stable drops is not connected, although the family of uninflected drops is. This has not been observed numerically, however.

\section{REFERENCES}

[1] ATHANASSENAS, M.: A variational problem for constant mean curvature surfaces with free boundary. J. Math. 377 (1987), 97 - 107.

[2] BirkHOFF, G., and G.C. RotA: Ordinary Differential Equations. 3rd ed. New York: John Wiley \& Sons 1978.

[3] CARTER. W. C.: The forces and behavior of fluids constrained by solids. Acta Metall 36 (1988), $2283-2292$.

[4] DELAUNAY, D. E.: Sur la surface dé révolution dont la courbure est constante. J. Math. Pures Appl. 6 (1941), 309 - 315.

[5] FINN, R.: Equilibrium Capillary Surfaces. New York: Springer-Verlag 1986.

[6] FINN. R.: Nonuniqueness and uniqueness of capillary surfaces. Man. Math. 61 (1988), $347-372$.

[7] JAHNKE, E., and F. EMDE: Table of Functions with Formulae and Curves. New York: Dover Publ. 1945.

[8] LANGBEIN, D.: Stability of liquid bridges between parallel plates. Preprint.

[9] RヘYLEIGH, J. W. S.: On the capillary phenomena of jets. In: Scientific Papers, Vol. 1. Cambridge: University Press 1899, pp. $433-442$.

[10] VOGEL, T. I.: Numerical results on trapped drop stability. Lawrence Berkeley Laboratories technical report, March 1991

[11] VOGEL. T.I.: Stability of a liquid drop trapped between two parallel planes. SIAM J. Appl. Math. 47 (1987), 516 - 525

[12] VOGEL, T. I.: Stability of a liquid drop trapped between two parallel planes II. General contact angles. SIAM J. Appl. Math. 49 (1989), $1009-1028$.

Received 18.06.1991

Prof. Dr. Robert Finn

Prof. Dr. T. I. Vogel

Department of Mathematics

Department of Mathematics

Stanford University

Texas A \& $M$ University

Stanford, CA 94305, USA

College Station, TX 77843 ,USA

\section{Book review}

L. de Branges, I. Gohberg and J. Roviryak (eds): Topics in Operator Theory - Ernst D. Hellinger Memorial Volume (Operator Theory: Advances and Applications: Vol. 48). Basel Boston - Berlin: Birkhäuser Verlag 1990; 448 pp.

The series "Operator Theory: Advances and Applications" edited by Israel Gohberg belongs to the most remarkable editions in the mathematical literature of the last decade. Since the beginning in 1979 up to now more than 50 volumes have been published. The main aims of this series include not only publishing recent progress in operator theory and related topics but also cultivating traditions and remembering the very roots of operator theory.

After the volumes 4 and 18 in this series which were dedicated to Otto Toeplitz and Issai Schur, respectively, there has been published a volume in honour of Ernst Hellinger who can be conceived as one of the founders of operator theory. Hellinger was among the first to demonstrate the potential of Hilbert's program in analysis, and he championed Hilbert's point of view 